\section{Magnetic resonance imaging and computed tomography of neurocysticercosis}

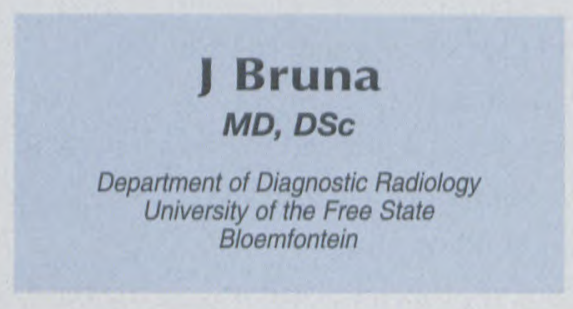

\section{Abstract}

The typical radiological appearance of neurocysticercosis (NC) includes: small solitary or multiple non-enhancing lesions, small enhancing nodules, non-enhancing cysts without and at a later stage with perifocal oedema, racemose cysts in the subarachnoid space, cysts with ring enhancement and perifocal oedema, cysts with eccentric scolex (target sign, bull's eye sign) and nodular calcifications. MRI is more sensitive than CT (Figs 1 - 5) in the diagnosis of subarachnoid and ventricular forms of NC. CT is very sensitive in diagnosing cerebral forms and is more sensitive than MRI in the late stage of NC characterised by nodular calcifications. In patients with seizures, neurological deficit and mental deterioration CT remains the imaging method of choice. If the result of $\mathrm{CT}$ is negative or dubious MRI has to be considered. On CT small lesions are not visible especially if investigation is performed only with $10 \mathrm{~mm}$ slices.

Neurocysticercosis (NC) may result from ingestion of food contaminated with Taenia solium eggs (ova) by the faeco-oral route or rarely by regurgitation (duodeno-gastric reflux) of Taenia solium eggs from the intestine into the stomach. The eggs are released from their encasement by gastric acid and 'hatch' in the intestinal mucus, producing oncospheres which are the primary larvae. The larvae penetrate the mucus and are distributed (disseminated) into the skin, muscles, fat tissues, bones and the central nervous system (CNS). Humans are the only known host of both the adult and larval forms of Taenia solium. The latter results in cysticercosis and later in taeniasis. NC is endemic in Africa, Asia, Central and South America. As a result of increased travel to the endemic areas and immigration from these countries NC has also been reported in Europe and North America. ${ }^{9,12,14,22}$ The role of computed tomography (CT) and magnetic resonance imaging (MRI) are well established in the diagnosis and monitoring of treatment of NC. ${ }^{1-26}$ The question remains whether both methods have the same value, and which of them is preferred. There are also different views on direct and indirect signs and on classification of NC. ${ }^{6,8,10,12,13,13,15-17,19,23,26}$ I would like to summarise our experience with $\mathrm{CT}$ and MRI of NC and to mention some differential diagnostic imaging characteristics according to my own experience.

\section{Material and methods}

The images of a randomised group of 110 patients with different stages of NC were re-evaluated. CT investigation was performed mainly by Somatom DR3 without and after intravenous administration of $50 \mathrm{ml}$ non-ionic contrast agent. Thickness of slices was $5-10 \mathrm{~mm}$, and steps also $5-10 \mathrm{~mm}$. Twenty-two patients were investigated by MRI (Magnetom Impact, 1 Tesla) without and after intravenous Gd-DTPA application (1 $\mathrm{mmol} / \mathrm{kg}$ ). Routine protocols of brain investigation were used: MRI-TISE sagittal and T2SE axial slices ( $5 \mathrm{~mm}$ ), and CT $5 \mathrm{~mm}$ slices of the posterior and $10 \mathrm{~mm}$ slices of the supratentorial region were used. The main clinical manifestations of $\mathrm{NC}$ in our patients are summarised in Table I. We re-evaluated number, size, localisation, density/intensity of lesions as well as indirect signs of NC (hydrocephalus, cerebral infarction, cortical atrophy and mass effect). According to our experience we summarised differential diagnostic signs and our approach in evaluation of NC.

\section{Results}

The radiological findings are summarised in Table II. There were 59 patients $(53.6 \%)$ with NC suffering from seizure, $35(31.8 \%)$ had neuro- 


\section{,}




\begin{tabular}{|lcc|}
\hline \multicolumn{3}{c|}{ Table I. Main clinical signs (no. of patients = 110) } \\
\hline Clinical sign & No. of patients & $\%$ \\
\hline Seizure & 59 & 53.6 \\
Neurological deficit & 35 & 31.8 \\
Mental deterioration & 10 & 9.0 \\
Intracranial hypertension & 4 & 3.6 \\
Headache & 2 & 1.8 \\
\hline
\end{tabular}

\begin{tabular}{|lcc|}
\hline \multicolumn{3}{|c|}{ Table II. MRI and CT findings (no. of patients = 110) } \\
\hline & No. of patients & $\%$ \\
\hline Cysts only & 26 & 23.6 \\
Nodule only & 8 & 7.2 \\
Cysts + ring enhancement & 24 & 21.8 \\
Calcifications + cysts + ring & 31 & 28.1 \\
Calcifications only & 34 & 30.9 \\
Hydrocephalus + NC signs & 12 & 10.9 \\
Cortical atrophy & 9 & 8.2 \\
Multiple & 96 & 87.3 \\
Diameter of lesions 1 - 10 mm & 99 & 70 \\
Localisation sub-/cortical & 77 & 24.5 \\
Subarachnoid + ventricular & 27 & \\
\hline
\end{tabular}

logical deficit and $10(9 \%)$ had different mental disturbances. The lesions were typically multiple in $96 / 110$ patients $(87.3 \%)$, with the number of lesions ranging from 2 to 500 in our group. Intracranial cysticercus, in the ganglia, and in the spinal canal was localised mainly subcortically or cortically and supratentorially (Figs 1 - 9). Occasionally NC was localised periand paraventricularly, subarachnoidally, intraventricularly (Figs 10 and 11), in the basal ganglia (Figs 12 and 13), and in the spinal canal, spinal cord (Figs 14 - 17), spinal subarachnoid as well as subdural spaces. Localisation simultaneously in different structures and spaces was also found. The diameter of the lesions was usually under $10 \mathrm{~mm}$ in 99/110 patients (90\%). MRI and CT images

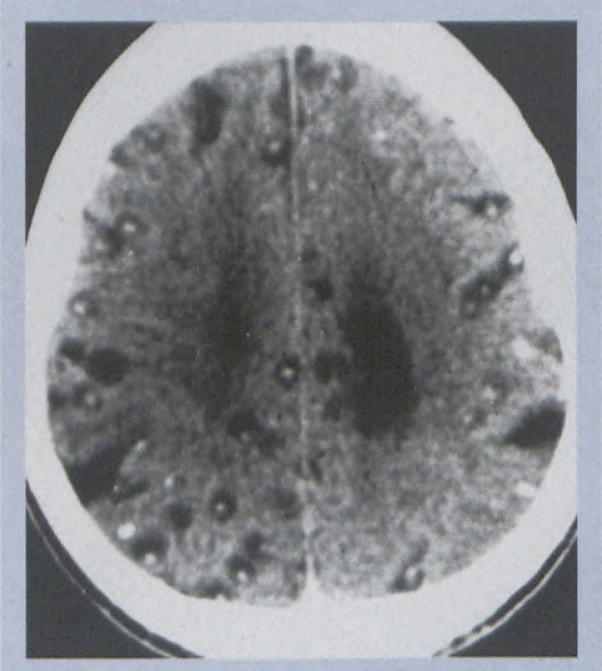

Fig. 1. Post-contrast CT image of multiple cystic lesions with scolices and cortical calcifications. No pathological enhancement. Stages 2 and 5 of NC (TS 18 yrs M).

of NC were usually those of small enhancing nodules, multiple small cysts with eccentric nodule (target or bull's eye sign) and ring enhancement.

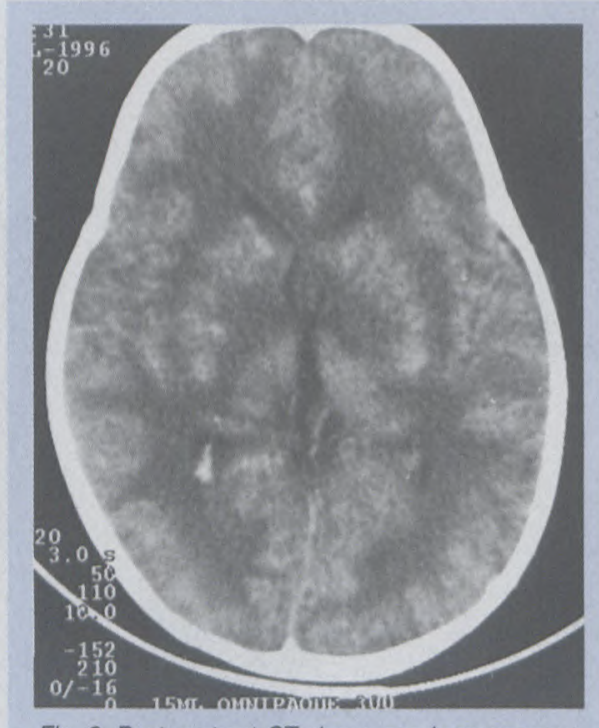

Fig. 2. Post-contrast CT shows non-homogeneous patchy enhancement of the basal ganglia and cortex. Stage 1 of NC (PZ2 yrs M).

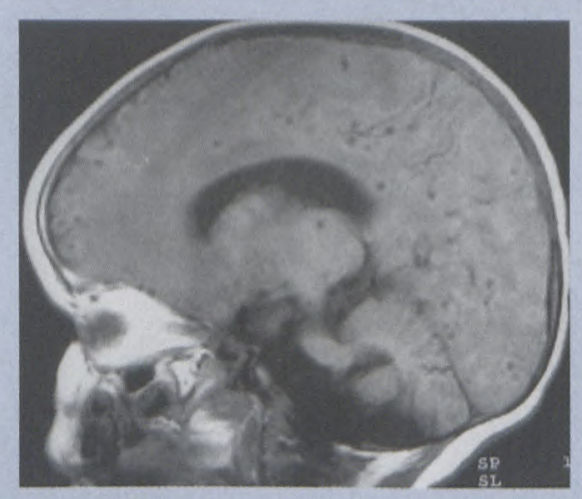

Fig. 3. T1WI shows multiple hypointense lesions with hyperintense surface. (PZ2 yrs $M$ ).

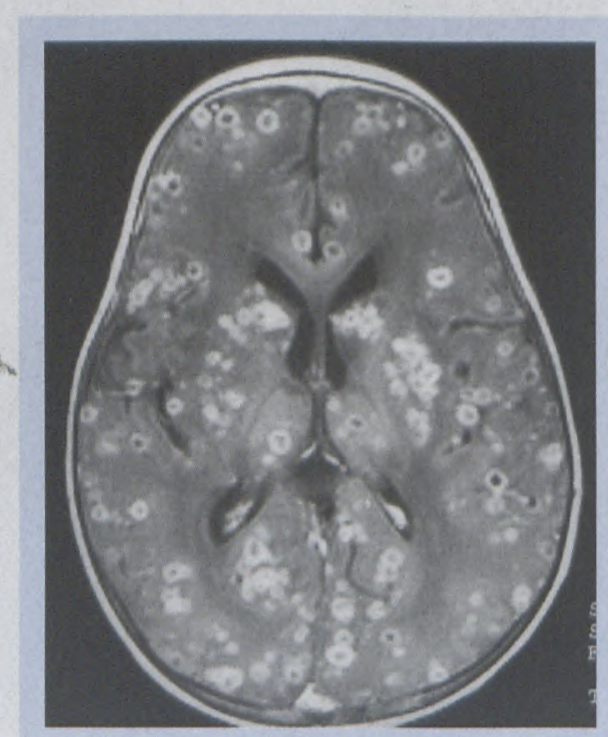

Fig. 4. T1WI post Gd-DTPA demonstrates multiple small enhancing lesions. Localisation of lesions: basal ganglia, thalamus, cortex and subcortex (PZ2 yrs M). 


\section{ORIGINAL ARTICLE}

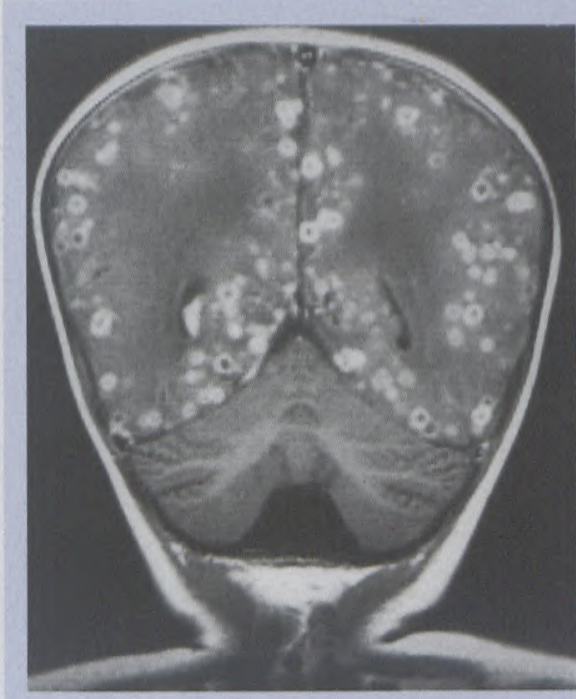

Fig. 5. Coronal T1WI post Gd-DTPA shows multiple enhancing lesions cortically and subcortically. Lesions are solid and some of them cystic stage 1 . 2 of NC (PZ 2 yrs M).

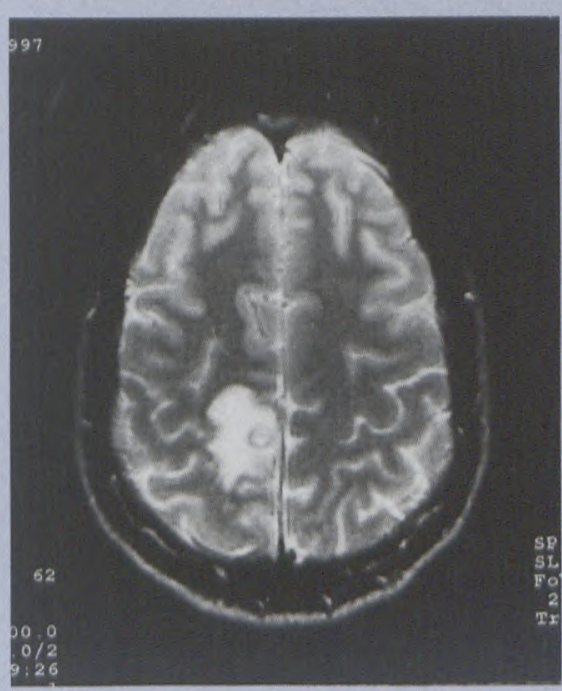

Fig. 6. Axial T2WI shows round hyperintense lesion with hypointense surface and hypointense area inside. Marked perifocal oedema (MA 19 yrs M).

Active lesions in the early dying stage had perifocal oedema. Scolices on T1W1 were often visible (14/22). Subarachnoid and intracerebral cysts appeared on CT and MRI without an evident intracystic eccentric nodule (scolex). The cyst capsule was iso- or hyperintense on T2WI. Double signal with some sediment was visible in large cysts. The largest intraventricular cyst in our group was $4 \mathrm{~cm}$ in diame-

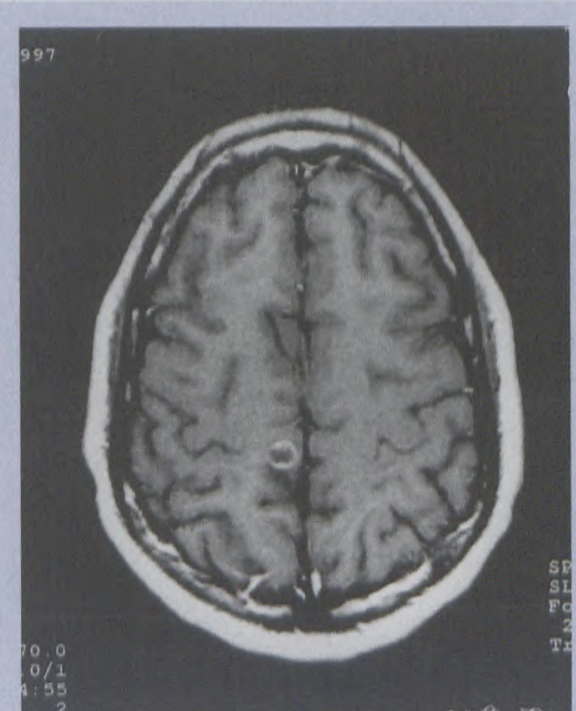

Fig. 7 Axial T1WI post Gd-DTPA shows ring enhancement and perifocal oedema. Stage 3 of NC (MA 19 yrs M).

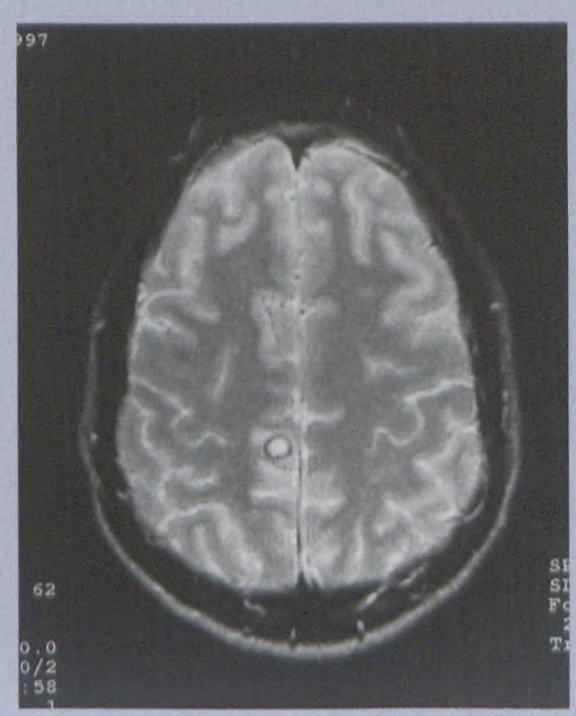

Fig. 8. Axial T2WI six weeks later shows hypointense lesion with hypointense capsule and small central hypointensity (MA 19 yrs M)

ter, the largest intracerebral cyst $3 \mathrm{~cm}$, and the largest subarachnoid $2 \mathrm{~cm}$. Very small lesions were not visible on CT but only on MRI. Some small calcifications were only visible on $\mathrm{CT}$. Different stages of NC were often visible simultaneously and were better differentiated by MRI (9/22).

\section{Discussion}

The clinical picture of NC varies

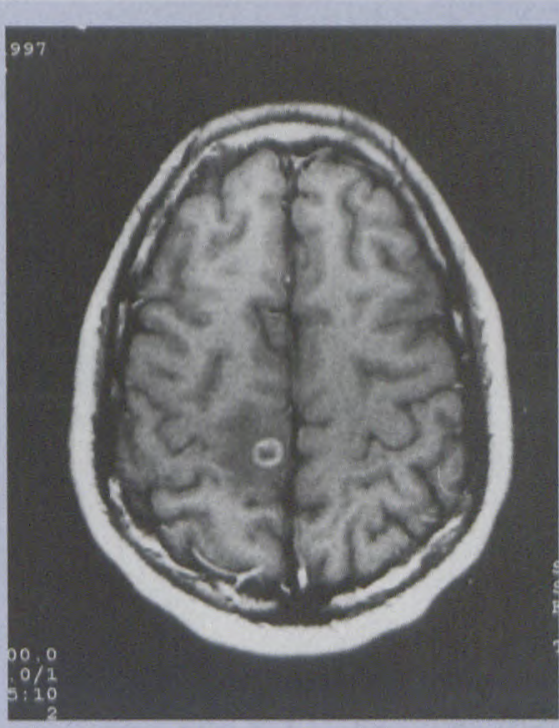

Fig. 9. Axial T1WI post GD-DTPA shows cortical cystic lesion with ring enhancement. Stage 4 of NC (MA 19 yrs M).

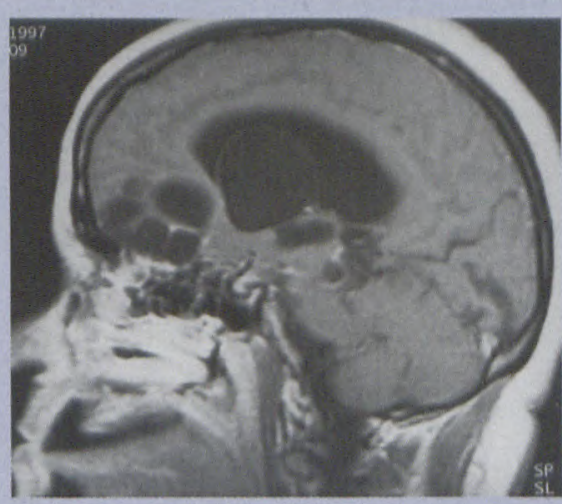

Fig. 10. Sagittal T1WI shows racemose cystic lesions sub-frontally, cystic lesion in frontal lobe and a large cyst in lateral ventricle near foramen of Monro causing obstructive hydrocephalus (RJ 37 yrs F).

from mild headache and neurological deficits to seizures, intracranial hypertension, and coma and death. ${ }^{4}$ Most patients with chronic NC presented with seizures and some neurological deficit. The acute stage (dissemination) as well as the vesicular stage are usually not diagnosed correctly because the accompanying symptoms are often only flu-like. ${ }^{15,16}$ Seizures are presenting symptoms of brain and meningeal lesions usually in advanced stage of the disease. Single brain lesions follow a relatively benign 


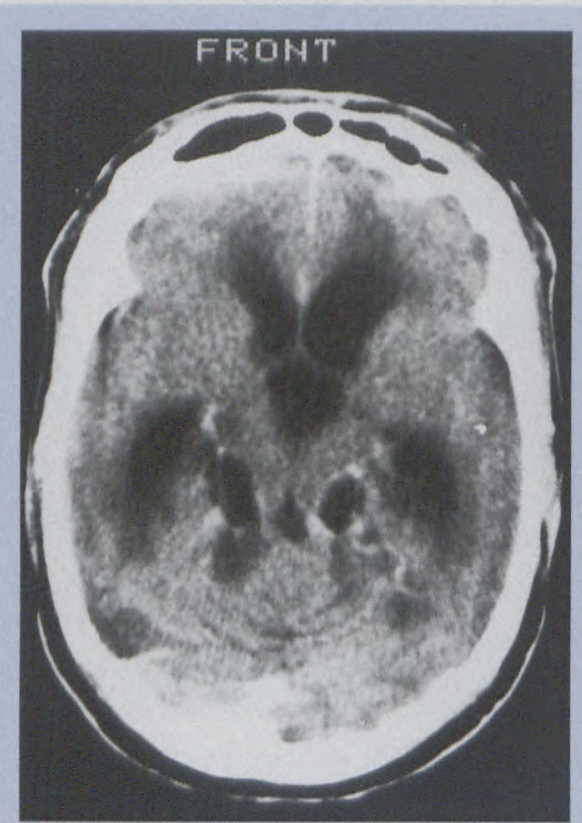

Fig. 11. Post-contrast CT shows an obstructive hydrocephalus and cystic lesion parasagittally and subarachnoidally (FN 33 yrs M).

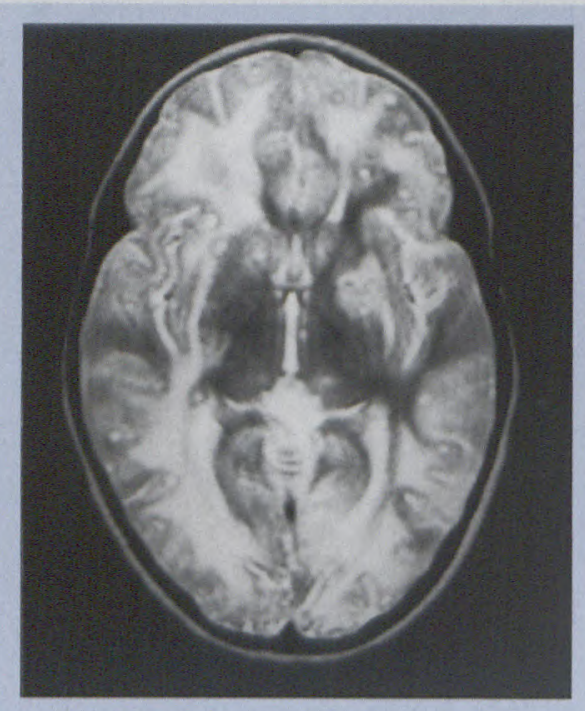

Fig. 12. Axial T2WI shows round small hyperintense lesions cortically, in basal ganglia and prominent white matter oedema which can correspond to leucoencephalitis (RM 8 yrs F).

course. ${ }^{4}$ Cerebrovascular ischaemic changes and lacunar infarcts in areas supplied by lenticulostriate arteries due to vasculitis have been reported. ${ }^{3,5,10}$ According to our experience the basal ganglia infarction is typical for neurotuberculosis. NC is classified into parenchymal, ventricular, meningeal, spinal and mixed forms. ${ }^{4,15}$

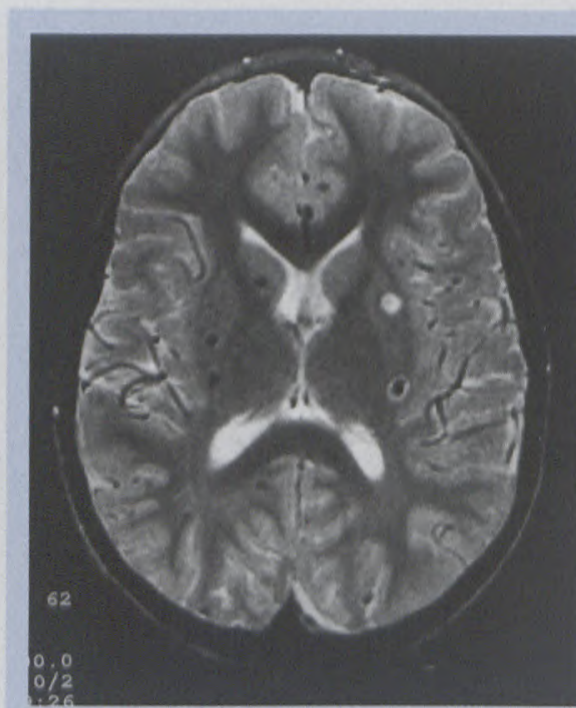

Fig. 13. Axial T2WI shows multiple hypointense lesions cortically and in basal ganglia. Some lesions have hyperintense surface due to perifocal oedema different stage of granuloma maturity (PJS 7 yrs M).

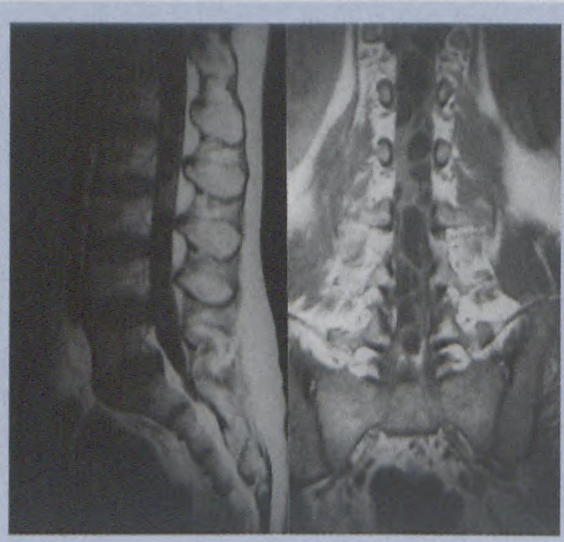

Figs 14 and 15. Sagittal and coronal TIWI show irregular round hypointense lesions involving subarachnoid space (JM 40 yrs M).

According to our study and literature data the parenchymal form is the most common. We can confirm that the radiological signs varied from focal non-enhancing areas of oedema, homogeneously enhancing lesions, non-enhancing cysts without and later with perifocal oedema, cysts with ring enhancement without and later with oedema and eventually calcifications. ${ }^{10,15,26}$ The indirect radiological signs of NC are hydrocephalus, cortical atrophy and rarely cerebral infarction or mass effect. ${ }^{16}$ The mass effect

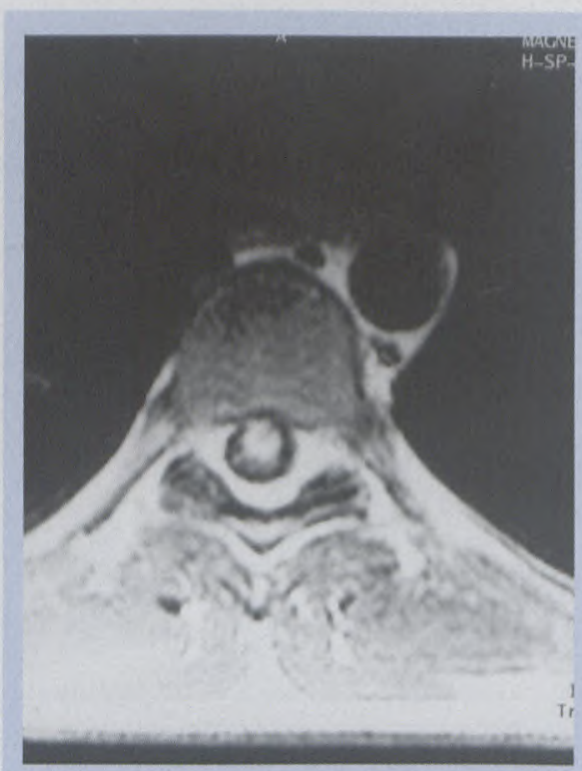

Fig. 16. Axial T1WI post Gd-DTPA shows large enhancing lesion localised in the intramedullary (PJ 25 yrs M).

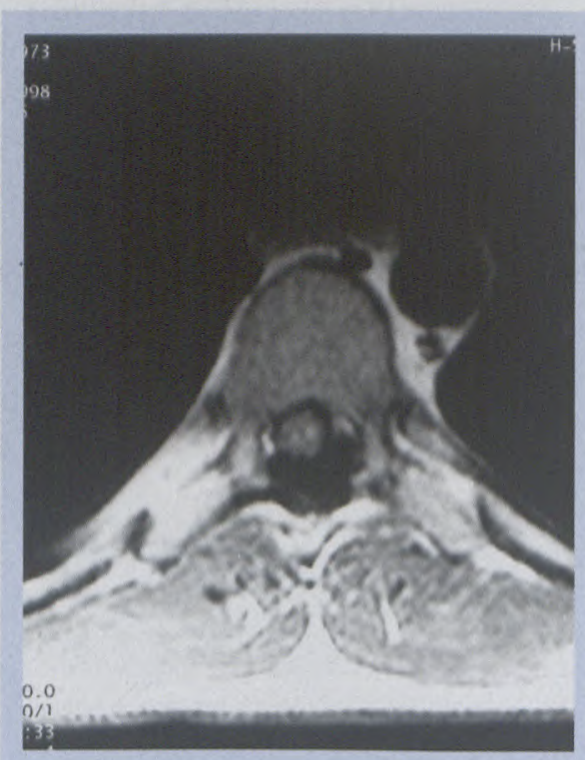

Fig. 17. Axial T1WI post GD-DTPA shows two small enhancing lesions of the spinal cord and large cystic extramedullar mass localised dorsally and proved subdurally (PJ 25 yrs M).

was only significant in all cases of spinal canal involvement. A typical imaging sign of $\mathrm{NC}$ is a cyst with an eccentric hyperdense small lesion localised inside on the wall of the cyst, which corresponds to scolex. This is not correctly called a target or bull's eye sign. With regard to classification, the first stage of NC is acute phase tis- 
sue invasion according to Kramer et $a l^{15}$ The first stage is well tolerated. The next vesicular stage with nonenhancing cysts starts in the chronic phase and is well tolerated, too. Clinical problems start when viable larvae in the cyst die. This first stage of degeneration or dying of the NC is characterised by marked perifocal inflammatory reaction; on both CT and MRI it presents as cysts with ring enhancement. With a dead larva the fluid in the cyst becomes turbid (colloid vesicular stage). On CT the cystic lesion has higher density and on MRI $\mathrm{T} 2 \mathrm{~W}$ it is hyperintense with a lower intensity ring. In the subsequent granulo-nodular stage the cyst shrinks, the cyst membrane becomes thicker and scolex calcifies. On both CT and MRI the dead cyst is isodense/isointense with brain tissue but on MRI there may be fine ring enhancement or rim hyperintensity representing residual perifocal inflammation with oedema. ${ }^{1724}$ The final stage is nodular or residual calcification. According to references and also to our experience, MRI is more sensitive than CT in diagnosing perifocal oedema and in differentiating subarachnoid NC from subarachnoid cysts. ${ }^{12,13,17,18}$ The cysts are found primarily in the cerebral grey matter and it is difficult to distinguish them from subarachnoid intercortical localisation within sulci. We also confirmed that MRI is more sensitive than $\mathrm{CT}$ in diagnosing the subarachnoid and ventricular forms. ${ }^{1,27} \mathrm{~A}$ non-calcified scolex is also more easily detected by MRI than by CT. ${ }^{10,17,19}$ The intensity of the signal of most parenchymal and intraventricular or meningeal cysts presumed to be viable is similar to that of CSF on both $\mathrm{T} 1$ and $\mathrm{T} 2$-weighted images. The cysts presumably degenerated have

\begin{tabular}{|c|c|c|c|}
\hline \multirow{3}{*}{$\begin{array}{l}\text { Acute phase } \\
\text { Chronic phase }\end{array}$} & \multicolumn{3}{|c|}{ Table III. MRI and CT images and morphology of NC } \\
\hline & Invasion & $\begin{array}{l}\text { Small non-enhancing lesion or } \\
\text { Small enhancing lesion with oedema }\end{array}$ & Stage 1 \\
\hline & Vesicular & $\begin{array}{l}\text { Non-enhancing cyst with scolex or } \\
\text { Cyst with ring enhancement }\end{array}$ & Stage 2 \\
\hline & Colloidal & $\begin{array}{l}\text { Cyst with ring enhancement + oedema } \\
\text { (white target on TIWI) }\end{array}$ & Stage 3 \\
\hline & Granular & $\begin{array}{l}\text { Shrunk enhancing cyst with } \\
\text { calcified scolex }\end{array}$ & Stage 4 \\
\hline & Nodular calcified & Calcified nodule 1 - $6 \mathrm{~mm}$ in diameter & Stage 5 \\
\hline
\end{tabular}

increased signal intensity on T1WI, probably because of increased protein content. ${ }^{24}$ Perifocal (pericystic) high signal intensity on both proton and $\mathrm{T} 2 \mathrm{WI}$ is a sign of the dying phase and represents inflammatory reaction caused initially by oedema and later by gliosis. Sensitivity of CT in diagnosis of NC is usually lower because of thicker slices routinely used for brain investigation. Thin slice $(2-5 \mathrm{~mm})$ CT can be a cost-effective alternative to MRI. ${ }^{19} \mathrm{CT}$ more easily detects the residual calcification and calcified scolex. More then $60 \%$ of our patients with $\mathrm{NC}$ already had calcifications by the time of first investigation. CT investigation is therefore the method of choice in patients with seizures or epilepsy of unknown origin with suspected NC. Seizures, neurological deficit and mental disturbances are the main clinical signs of brain involvement. ${ }^{11,20,22}$ In the differential diagnosis of $\mathrm{NC}$ it is necessary to exclude tuberculosis, toxoplasmosis, abscesses, malignancy and paragonimiasis. ${ }^{16}$ All of them may give a slightly similar imaging pattern. The imaging signs, morphology and classification of NC according to the literature $^{6,15}$ as well as the main imaging signs are summarised in Table III. The main differential imaging signs of $\mathrm{NC}$ and neurotuberculosis, abscess, toxo- plasmosis and malignancy are summarised in Table IV.

\section{Acknowledgements}

Acknowledgements to Professors J Albertyn, J F K de Villiers, A J Kruger and Dr F Haven for submission of certain clinical data.

\section{References}

1. Barloom TJ, Yuh WT, Chiang FL. Lesions involving the fourth ventricle evaluated by $\mathrm{CT}$ and MT. Magn Reson Imaging 1989; 7: 635-642.

2. Boecher-Scwertz HG, Hey O, Higer HP, Perneczky A. Intrasellar cysticercosis mimicking a pituitary adenoma. Br J Neurosurg 1991; 5: 405-407.

3. Bruna J, de Villiers JFK, Haven F, Albertyn J. Magnetic resonance imaging and computed tomography of neurocysticercosis. MAGMA 1998; 6: suppl 1, 185.

4. Brutto del OH. Cysticercosis and cerebravascular disease. J Neurol Neurosurg Psychiatry 1992; 55: 252-254.

5. Cantu C, Barinagarrementeria F. Cerebrovascular complications of neurocysticercosis. Arch Neurol 1996; 53: 233-239.

6. Carbajal JR, Palacios E, Azar-kia B, Churchill R. Radiology of cysticercosis of the central nervous system including computed tomography. Radiology 1977; 1: 127-131.

7. Celis MA, Mourier KL, Polivka M. Cysticercose cisternale de l'angle ponto-cerebelleoux. Neurochirurgie 1992; 38: 108-112.

8. Chang $\mathrm{KH}$, Lee JH, Hann MH, Han MC. The role of contrast-enhanced MRI in the diagnosis of neurocysticercosis. Am J Neuroradiol 1991; 12: 509-512.

9. Cueter AC, Gracia-Bobadile J, Guerra LG, Martinez FM, Kairn B. Neurocysticercosis: focus on intraventricular disease. Clin Infect Dis 1997; 24: 157-164.

10. Dumas JL, Visy JM, Belin C, et al. Paremchymal neurocysticercosis: follow-up and staging by MRI. Neuroradiology 1997; 39: 12-18.

11. Escobedo F, Sotelo J, Penagos P, Rodriquez J, del Brutto O. Albendazole therapy for neurocysticercosis, a controlled study with CT and MR. Acta Leiden 1989; 57: 247-254. 


\section{ORIGINAL ARTICLE}

Table IV. General differential imaging characteristics of NC

\begin{tabular}{llllll}
\hline & NC & TB & Abscess & Toxoplasmosis & TU-METS \\
\hline Number of lesions & $>10$ & $<10$ & $1-3$ & $5-10$ & $1-$ Multi \\
Size (mm) & $<10$ & $>10$ & $>10$ & $>10$ & $>10$ \\
Localisation & Cortical & White matter & WM & WM & WM \\
Multiloculation & Occasional & Occasional & Occasional & No & Occasional \\
Ring enhancement & Yes & Yes & Thick & Yes & Irregular \\
Density (HU) & $1-10$ & $20-30$ & $15-20$ & $20-30$ & Hyper \\
T2WI & Hyper & Hypo & Hyper & Hyper & Hyper \\
Surface of lesion & Round & Round & Round & Irregular & Round/irregular \\
Calcifications & Stage $4-5$ & Healed & No & Healed & Occasional \\
HU = Haunsfield unit. & & & & &
\end{tabular}

12. Ginier BL, Poirier VC. MRI of intraventricular cysticercosis. Am J Neuroradiol 1992; 13: 1247 1248.

13. Gulati P, Jena A, Tripathi RP, Gupta AK. MRI in childhood epilepsy. Indian J Pediatr 1991; 28: 761-765.

14. Hansen NJD, Hagelskjaer LH, Christtensen T. Neurocysticercosis. Scand J Infect Dis 1992; 24: 255-262.

15. Kramer LD, Locke GE, Byrd SE, Daryabagi I Cerebral cysticercosis. Radiology 1989; 171:459469.

16. Michael AS, Levy JM, Paige ML. Cysticercosis mimiking brain neoplasm. I Computed Assist Tomogr 1990; 14: 708-711.

17. Palacios E, Lujambio PS, Jasso RR. Computed tomography and magnetic resonance imaging of neurocysticercosis (Review). Semin Roentgenol 1997; 4: 325-334.

18. Puri V, Gupta RK. MRI evaluation of focal com puted tomography abnormality in epilepsy. Epilepsia 1991; 32: 460-466.

19. Rajshekhar V, Chandy MJ. Comparative study of CT and MRI in patients with seizures and a solitary cerebral cysticercus granuloma. Neuroradiology 1996; 38: 542-546.

20. Robles C, Sedano AM, Vargas-Tentori N Galindo-Vigen S. Long term results of praziquantel therapy in neurocysticercosis. I Neurosurg 1987; 66: 359-363.

21. Rosenfeld EA, Byrd SE, Shulman ST. Neurocysticercosis among children in Chicago. Clin Infect Dis 1996; 23: 262-268.
22. Shriqui CL, Milette PC. You drive me crazy: Acute psychosis and neurocysticercosis. Can J Psychiatry 1992; 37: 120-124.

23. Sotelo J, Marin C. Hydrocephalus secondary to cysticerosis arachnoiditis. J Neurosurg 1987; 66: 686-689.

24. Teilbaum GP, Otto RJ, Lin M, et al. MR imaging of neurocysticercosis. Am J Roentgenol 1989; 153: $857-866$

25. Ter Pennig B, Litchman CD, Heier L. Bilatera middle cerebral artery occlusions in neurocysticercosis. Stroke 1992; 23: 280-283

26. Zee $\mathrm{C}$, Segall $\mathrm{HD}$, et al. Magnetic resonance imaging of neurocysticercosis. I Comput Assist Tomogr 1988; 12: 927-934. 